\title{
WD0433+270: an old Hyades stream member or an Fe-core white dwarf? ${ }^{\star}$
}

\author{
S. Catalán ${ }^{1,2}$, I. Ribas ${ }^{1,2}$, J. Isern ${ }^{1,2}$, and E. García-Berro ${ }^{1,3}$ \\ 1 Institut d'Estudis Espacials de Catalunya, c/ Gran Capità 2-4, 08034 Barcelona, Spain \\ e-mail: catalan@ieec.uab.es \\ 2 Institut de Ciències de l'Espai, CSIC, Facultat de Ciències, Campus UAB, 08193 Bellaterra, Spain \\ 3 Departament de Física Aplicada, Escola Politècnica Superior de Castelldefels, Universitat Politècnica de Catalunya, \\ Avda. del Canal Olímpic s/n, 08860 Castelldefels, Spain
}

Received 5 July 2007 / Accepted 15 October 2007

\begin{abstract}
Context. G39-27/289 is a common proper-motion pair formed by a white dwarf (WD0433+270) and a main-sequence star (BD+26 730) that apparently has been classified as a member of the Hyades open cluster. Previous studies of the white dwarf component yielded a cooling time of $\sim 4 \mathrm{Gyr}$. Although it has not been pointed out before explicitly, this result is 6 times greater than the age of the Hyades cluster, giving rise to an apparent conflict between the physics of white dwarfs and cluster main-sequence fitting.

Aims. We investigate whether this system belongs to the Hyades cluster and, accordingly, give a plausible explanation for the nature of the white dwarf member.

Methods. We have performed and analyzed spectroscopic observations to better characterize these objects, and used their kinematic properties to evaluate their membership in the Hyades. Then, different mass-radius relations and cooling sequences for different core compositions ( $\mathrm{He}, \mathrm{C} / \mathrm{O}, \mathrm{O} / \mathrm{Ne}$ and $\mathrm{Fe}$ ) were employed to infer the mass and cooling time of the white dwarf.

Results. From kinematic and chemical composition considerations, we believe that the system was a former member of the Hyades cluster and therefore has an evolutionary link with it. However, the evidence is not conclusive. With regards to the nature of the white dwarf component, we find that two core compositions $-\mathrm{C} / \mathrm{O}$ and $\mathrm{Fe}-$ are compatible with the observed effective temperature and radius. These compositions yield very different cooling times of $\sim 4 \mathrm{Gyr}$ and $\sim 1 \mathrm{Gyr}$, respectively.

Conclusions. We distinguish two possible scenarios. If the pair does not belong to the Hyades cluster but only to the Hyades stream, this would indicate that such a stream contains rather old objects and is definitely not coeval with the cluster. This has interesting consequences for Galactic dynamics. However, our favored scenario is that of a white dwarf with a rather exotic Fe core, having a cooling time compatible with the Hyades age. This is a tantalizing result that would have implications for the thermonuclear explosion of white dwarfs and explosion theories of degenerate nuclei.
\end{abstract}

Key words. stars: evolution - stars: white dwarfs - Galaxy: open clusters and associations: individual: WD0433+270 stars: kinematics

\section{Introduction}

The determination of reliable ages is of obvious importance to both astrophysics and cosmology, but it is not exempt from many complications - see, e.g., von Hippel et al. (2001). The age of a star is perhaps the most difficult property to estimate and it nearly always depends on rather strong model assumptions (Mamajek et al. 2007). An exception is the method that uses radioactive decay to estimate stellar ages directly (Cayrel et al. 2001; Frebel et al. 2007), but this has rather restricted applicability. Another emerging technique is asteroseismology, which has the potential of providing accurate ages (Miglio \& Montalbán 2005), but this will require high-precision photometric data from upcoming space missions and also stars with wellconstrained physical properties. In the meantime, the use of open clusters and main sequence stellar evolutionary models continues to be the most widely used method to infer the ages of stars in the Galaxy. In spite of the still-present uncertainties, such as convective overshoot, chemical composition anomalies, etc., the

* Based on observations obtained at: Calar Alto Observatory, Almería, Spain and McDonald Observatory, Texas, USA. field is mature enough to provide ages that are reliable to better than $\sim 10 \%$ - see, for instance, Paunzen \& Netopil (2006). The analogous method of using eclipsing binaries (Ribas et al. 2000) yields similar (model-dependent) accuracy.

The study of white dwarfs has made very valuable contributions to numerous areas of astrophysics, and estimating individual ages of stars is no exception. The main advantage of white dwarfs is the conceptual simplicity of their evolution, which can be described as a simple cooling process. Modeling the cooling sequences makes white dwarfs powerful stellar chronometers, accurate to about 25\% (Silvestri et al. 2005). Among the major uncertainties of this method are the pre-white dwarf evolution time and the effects of the chemical composition of the core.

In this paper we discuss a case that presents an interesting puzzle in which the age estimate stemming from the white dwarf cooling sequence apparently conflicts with the age estimate coming from cluster membership. The object is G39-27/289, a common proper motion pair formed by a DA white dwarf (WD0433+270) and a K type star - BD+26 730 (Holberg et al. 2002) - which is a well-studied variable star (V833 Tau) and also a single-lined spectroscopic binary with a very low-mass 
Table 1. Photometric data and stellar parameters derived for BD+26 730 .

\begin{tabular}{|c|c|c|c|c|c|c|c|}
\hline$\overline{\bar{V}}$ & $\overline{J J}$ & $\bar{H}$ & $\overline{\bar{K}}$ & $\overline{T_{\text {eff }}(\mathrm{K})}$ & $\overline{[\mathrm{Fe} / \mathrm{H}]}$ & $\bar{Z}$ & $\bar{l} \log \left(L / L_{\odot}\right)$ \\
\hline $8.42 \pm 0.02$ & $5.945 \pm 0.023$ & $5.400 \pm 0.018$ & $5.240 \pm 0.023$ & $4,595 \pm 30$ & $0.03 \pm 0.09$ & $0.021 \pm 0.004$ & $-0.527 \pm 0.021$ \\
\hline
\end{tabular}

companion (Tokovinin et al. 2006). The members of a common proper motion pair were likely born simultaneously with the same chemical composition (Wegner 1973; Oswalt et al. 1988). Since the components are separated well ( $2200 \mathrm{AU}$ in this case), no mass exchange has taken place and they have evolved as isolated stars. Thus, it is logical to assume that both components of G39-27/289 have the same age and the same original metallicity. The $K$-star component and, by extension, its common proper motion companion were classified as Hyades members by Perryman et al. (1998) and therefore would have an age ranging from about 0.6 to 0.7 Gyr. However, this obviously conflicts with the cooling time of WD0433+270, which has been estimated to be about 4 Gyr (Bergeron et al. 2001).

In this work, we carry out a detailed study of these objects, using both information present in the literature and our own spectroscopic observations, with the objective of unveiling their nature and their possible membership in the Hyades open cluster. We evaluate the different scenarios and discuss their respective implications to white dwarf physics and Galactic dynamics.

\section{Observations}

We observed both components of the common proper motion pair, namely BD+26 730 and WD0433+270, with the objective of characterizing their radiative properties. In the case of BD+26 730, we performed spectroscopic observations with the FOCES echelle spectrograph on the $2.2 \mathrm{~m}$ telescope at CAHA (Almería, Spain), obtaining a resolution of $R \sim 47000$. The data were reduced following the standard procedures using the echelle tasks of the IRAF package. From these observations, together with a detailed study of the visible and near-infrared spectral energy distribution of the star, we derived the effective temperature and metallicity of $\mathrm{BD}+26730$ following the procedure described in Catalán et al. (2007). The resulting value of $T_{\mathrm{eff}}=4595 \pm 30 \mathrm{~K}$ is in good agreement with the determination of Oláh et al. (2001). The chemical composition analysis yielded a nearly solar metallicity of $[\mathrm{Fe} / \mathrm{H}]=0.03 \pm 0.09$. The total luminosity, $L$, was calculated from the apparent magnitude and a bolometric correction (Masana et al. 2006). A summary of the photometric information of BD+26 730 and the derived stellar parameters is given in Table 1.

A very relevant parameter for our study is the age of $\mathrm{BD}+26$ 730. Unfortunately, this cannot be determined reliably from the observed $T_{\text {eff }}$ and $L$ using model isochrones because of the relative proximity of the star to the zero-age main sequence. Note, however, that Barrado y Navascués \& Stauffer (1996) determined lithium abundances for a sample of binaries classified as Hyades members, including BD+26 730. Lithium was indeed detected in this case, obtaining a value of $\log N_{\mathrm{Li}}=$ 0.31 , which is in good accord with the rest of the observed binaries, albeit with an abundance somewhat larger than that of single objects belonging to the Hyades $\left(\log N_{\mathrm{Li}}=-0.5\right)$. According to Barrado y Navascués \& Stauffer (1996), this is something expected since, in general, binary systems have an overabundance of lithium with respect to single stars. Later, Barrado y Navascués et al. (1997) recalculated the lithium abundance of $\mathrm{BD}+26730$, obtaining in this case $\log N_{\mathrm{Li}}=0.23$. They compared the $\mathrm{Li}$ abundance and the effective temperature
Table 2. Photometric properties and parallax of WD0433+270.

\begin{tabular}{lccccccc}
\hline \hline$B^{1}$ & $V$ & $R$ & $I$ & $J$ & $H$ & $K$ & $\pi(\mathrm{mas})$ \\
\hline 16.48 & 15.81 & 15.40 & 15.01 & 14.61 & 14.42 & 14.32 & $60 \pm 3$ \\
\hline
\end{tabular}

${ }^{1}$ The uncertainties are typically $3 \%$ for $V, R$, and $I$, and $5 \%$ for the rest.

Table 3. Atmospheric parameters of WD $0433+270$ available in the literature.

\begin{tabular}{lcc}
\hline \hline$T_{\text {eff }}(\mathrm{K})$ & $\log g$ & Reference \\
\hline $5620 \pm 110$ & $8.14 \pm 0.07$ & Bergeron et al. (2001) \\
$5434 \pm 300$ & $8.0 \pm 0.1$ & Zuckerman et al. (2003) \\
\hline
\end{tabular}

of this object with lithium depletion isochrones, yielding an age of $\sim 600$ Myr. Thus, the results suggest a Hyades age for $\mathrm{BD}+26730$.

In the case of WD0433+270, a cool white dwarf, the best way to estimate its effective temperature is by using the photometric energy distribution instead of a spectroscopic fit (Bergeron et al. 2001). However, we decided to observe this object to ensure its spectral classification. The observations were performed with the LCS spectrograph of the Harlan J. Smith (2.7 m) telescope at McDonald Observatory (Texas, USA) covering some of the main Balmer lines (from 3885 to $5267 \AA$ ) and obtaining a resolving power of $\sim 5 \AA$ FWHM. We used the standard procedures within the single-slit tasks of the IRAF package. The spectrum shows weak absorption lines, but we were able to identify $\mathrm{H} \beta$ unambiguously. The weakness of the lines is the reason this star had been previously classified as a type DC white dwarf by several authors (Eggen \& Greenstein 1965; Oswalt \& Strunk 1994). Higher resolution observations by Bergeron et al. (2001) and Zuckerman et al. (2003) have permitted the identification of $\mathrm{H} \alpha$ and $\mathrm{H} \beta$, respectively. Putting all results together we can confidently classify this object as a DA white dwarf.

Bergeron et al. (2001) used BVRIJHK photometry - see Table 2 - to obtain the energy flux distribution of WD0433+270. From a fit of different theoretical energy distributions, they obtained its effective temperature and $(R / d)^{2}$, where $R$ is the radius of the star and $d$ the distance. The radius can be determined accurately because a trigonometric distance is available for this object. The authors also obtained its mass and $\log g$ from the mass-radius relation of Fontaine et al. (2001) for C/O white dwarfs. Zuckerman et al. (2003) followed the same procedure as Bergeron et al. (2001), but using the mass-radius relation of Wood (1995). The results by these authors are summarized in Table 3. It is worth mentioning that the spectroscopic observations were not used in any case to estimate the stellar properties, but they served as an internal check of the photometric solutions via comparison of calculated models with the observed line profiles.

The luminosity of the white dwarf can be derived from its effective temperature (Bergeron et al. 2001) and distance $(d=$ $16.95 \pm 0.86 \mathrm{pc}$ ), and considering its apparent magnitude and the bolometric corrections of Masana et al. (2006). The value that we obtained is $\log \left(L / L_{\odot}\right)=-3.92 \pm 0.04$ and, from this, 
Table 4. Spatial velocities for the studied stars and the Hyades cluster and streams.

\begin{tabular}{lcccccc}
\hline \hline & $\begin{array}{c}\mu_{\alpha} \\
(\mathrm{mas} / \mathrm{yr})\end{array}$ & $\begin{array}{c}\mu_{\delta} \\
(\mathrm{mas} / \mathrm{yr})\end{array}$ & $\begin{array}{c}V_{r} \\
\left(\mathrm{~km} \mathrm{~s}^{-1}\right)\end{array}$ & $\begin{array}{c}U \pm \sigma_{U} \\
\left(\mathrm{~km} \mathrm{~s}^{-1}\right)\end{array}$ & $\begin{array}{c}V \pm \sigma_{V} \\
\left(\mathrm{~km} \mathrm{~s}^{-1}\right)\end{array}$ & $\begin{array}{c}W \pm \sigma_{W} \\
\left(\mathrm{~km} \mathrm{~s}^{-1}\right)\end{array}$ \\
\hline WD0433+270 & 228 & -155 & +36.3 & -39.2 & -15.5 & -1.8 \\
WD0433+270 $^{2}$ & 228 & -155 & +41.7 & -44.4 & -15.7 & -3.9 \\
${\text { BD+26 } 730^{3}}^{3}$ & 232.36 & -147.11 & $+36.18 \pm 0.08$ & -39.4 & -17.2 & -1.6 \\
Hyades OCl $^{4}$ & $\ldots$ & $\ldots$ & $\ldots$ & $-42.8 \pm 3.6$ & $-17.9 \pm 3.2$ & $-2.2 \pm 5.2$ \\
${\text { Hyades SCl}(1)^{4}}^{4}$ & $\ldots$ & $\ldots$ & $\ldots$ & $-31.6 \pm 2.8$ & $-15.8 \pm 2.8$ & $0.8 \pm 2.7$ \\
${\text { Hyades SCl}(2)^{4}}_{\text {Hyades SCl}(3)^{4}}$ & $\ldots$ & $\ldots$ & $\ldots$ & $-33.0 \pm 4.2$ & $-14.1 \pm 4.0$ & $-5.1 \pm 3.1$ \\
Hyades Stream $^{5}$ & $\ldots$ & $\ldots$ & $\ldots$ & $-32.8 \pm 2.8$ & $-11.8 \pm 2.8$ & $-8.9 \pm 2.9$ \\
Hyadean
\end{tabular}

${ }^{1}$ Proper motions from Salim \& Gould (2003) and velocities from Zuckerman et al. (2003).

2 Proper motions from Salim \& Gould (2003) and velocities recalculated considering an Fe core (see Sect. 4).

${ }^{3}$ Spatial velocities calculated from the radial velocity and proper motions reported by Perryman et al. (1997).

${ }^{4}$ Spatial velocities from Chereul et al. (1999).

${ }^{5}$ Spatial velocities from Famaey et al. (2005).

a radius of $R=0.0115 \pm 0.0010 R_{\odot}$. Both results are in good agreement with those reported by Bergeron et al. (2001).

\section{Membership in the Hyades}

The membership of WD0433+270 in the Hyades cluster was evaluated by Eggen (1993a) when studying all degenerate stars with certain kinematic criteria. WD0433+270 was excluded from the member list, although the radial velocity of its companion is identical to the value predicted by membership. The justification was a color index supposedly too red to belong to the Hyades, but the distance of the common proper motion pair to the center of the cluster was more significant. This author concluded that this common proper motion pair is projected on the cluster but only at about a third of the distance to the cluster.

The comprehensive study of Perryman et al. (1998) considered the 5490 Hipparcos Catalog stars corresponding to the field of the Hyades. Hipparcos astrometry was combined with radial velocity measurements in order to obtain three-dimensional velocities, which allowed candidate membership selection based on position and kinematic criteria. The authors divided the Hyades into four components by using the three-dimensional distance to the cluster center. The distance of BD+26 730 to the center of the cluster is $29 \mathrm{pc}$ and therefore it was classified as a former member of the Hyades cluster, currently lying beyond the tidal radius $(\sim 10 \mathrm{pc})$. In contrast, de Bruijne (1999) preferred not to include $\mathrm{BD}+26730$ in their list of Hyades member stars following the conclusions of a study based on the convergent-point method.

It is also worth mentioning two other pieces of evidence that were not included in the studies mentioned above. One is the metal content of $\mathrm{BD}+26$ 730. As discussed in Sect. 2, we have reliably determined the metallicity of this star and obtained a value of $[\mathrm{Fe} / \mathrm{H}]=0.03 \pm 0.09$. This agrees reasonably well with the Hyades metallicity of $[\mathrm{Fe} / \mathrm{H}]=0.14 \pm 0.05$ as determined by Perryman et al. (1998). The result is not conclusive, however, because the mean metallicity of field stars is $[\mathrm{Fe} / \mathrm{H}]=$ $-0.14 \pm 0.19$ (Nordström et al. 2004). The other important point is the detection of lithium in the spectrum of $\mathrm{BD}+26730$ by Barrado y Navascués \& Stauffer (1996), which clearly favors a relatively young age for this object and agrees with the rest of the Hyades members studied.

It has often been mentioned that there is a spatially unbound group of stars in the solar neighborhood with the same kinematics as the Hyades open cluster (Eggen et al. 1993b;
Perryman et al. 1998). This group of stars is called the Hyades stream or Hyades supercluster. Chereul et al. (1999) have mapped the density-velocity inhomogeneities of an absolute magnitude limited sample of A-F type dwarfs. Three different clumps within the Hyades stream were distinguished, each one of them with characteristic space velocities, which are given in Table 4. The authors also claim that the Hyades stream probably contains three groups of $0.5-0.6 \mathrm{Gyr}, 1 \mathrm{Gyr}$, and 1.6-2 Gyr, which are in an advanced stage of dispersion in the same velocity volume. Each stream presents a characteristic age distribution, although the velocity separation does not produce a clear age separation.

In Table 4 we provide the radial and space velocities of $\mathrm{BD}+26730$ and WD0433+270. We also give the kinematic properties of the Hyades open cluster $(\mathrm{OCl})$ and of each clump within the stream according to Chereul et al. (1999) (called $\mathrm{SCl} 1,2$, and 3 by these authors). The recent spatial velocities of the Hyades stream calculated by Famaey et al. (2005) are also listed. As can be seen, both members of the common proper motion pair have velocities that are compatible with those of the Hyades open cluster and are somewhat different from the velocities characteristic of the Hyades stream or the clumps within the stream.

From these kinematic considerations, together with the lithium detection in $\mathrm{BD}+26730$, we favor the hypothesis that the common proper motion pair studied here is indeed linked to the Hyades cluster evolution-wise. It is certainly not a bona fide cluster member because of its location beyond the tidal radius of the cluster, but it is more likely a former member that has escaped. If this scenario is correct, the components of the pair should have the age of the Hyades cluster, which was estimated to be $625 \pm 50$ Myr by Perryman et al. (1998). However, we do not have conclusive evidence supporting this evolutionary link, and so the alternative scenario that this pair belongs to the Hyades stream cannot be completely ruled out.

\section{The nature of WD0433 +270}

Most white dwarfs have a core made of $\mathrm{C} / \mathrm{O}$, although other compositions are possible. However, in this particular case, and given the putative age discrepancy with the Hyades cluster, we consider all the feasible compositions that have been proposed to date. In Fig. 1 we show mass-radius relations considering different compositions for the core: He (Serenelli et al. 2002), $\mathrm{C} / \mathrm{O}$ (Salaris et al. 2000), O/Ne (Althaus et al. 2005), and $\mathrm{Fe}$ 


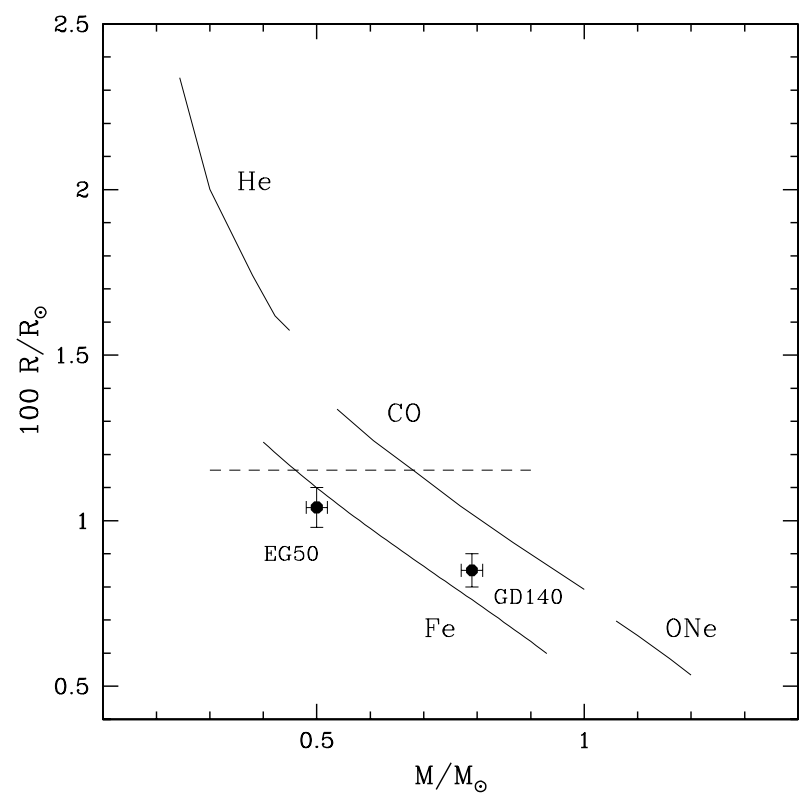

Fig. 1. Mass-radius relations for different compositions and $T_{\text {eff }}=$ $5500 \mathrm{~K}$. The dashed line corresponds to the radius of WD0433+270. The filled circles correspond to observational data obtained by Provencal et al. (1998).

(Panei et al. 2000a). All these relations correspond to an effective temperature of $5500 \mathrm{~K}$. As can be seen, the radius of WD0433+270 only has a corresponding mass in the case of $\mathrm{C} / \mathrm{O}$ and $\mathrm{Fe}$ cores. The existence of Fe white dwarfs has been pointed out in the past. Provencal et al. (1998) derived the radii of some field white dwarfs using parallaxes and effective temperatures. Then, they obtained the masses from the radii and surface gravities, which were previously determined independently from spectroscopic fits to white dwarf models. In two cases (GD 140 and EG 50), they obtained masses that were too small to fit the $\mathrm{C} / \mathrm{O}$ mass-radius relation. Their best explanation for these stars is that they have an Fe core (Shipman \& Provencal 1999). The data corresponding to these stars have also been plotted in Fig. 1.

The gravitational redshift and the radial velocity of a white dwarf are two interdependent parameters. Zuckerman et al. (2003) calculated the radial velocity of WD0433+270 (see Table 4) assuming a C/O core. Since an Fe core also seems possible in this case, we recalculated this value considering the massradius relation of Panei et al. (2000b) for Fe white dwarfs. As seen in Table 4, radial and space velocities are compatible in both cases with the kinematic properties of the Hyades cluster, considering the typical errors of white dwarf radial velocities (Schultz et al. 1996).

Turning the argument around, the kinematics can also be used to estimate the mass of WD0433+270 independently of the composition of the core. This can be done assuming that WD0433+270 has the same radial velocity as its companion, $\mathrm{BD}+26730$, and considering the observed velocity of this white dwarf reported by Zuckerman et al. (2003). This yields a mass of $\sim 0.55 M_{\odot}$, which does not conclusively favor any of the two core compositions $(\mathrm{C} / \mathrm{O}$ or $\mathrm{Fe})$.

Once we know the masses that correspond to each massradius relation (see Table 5), we can calculate the cooling times using the proper cooling sequences. In Fig. 2 we show the cooling sequences for different core compositions: C/O (topleft), O/Ne (top-right), $\mathrm{He}$ (bottom-left), and $\mathrm{Fe}$ (bottom-right). We have overplotted the effective temperature and luminosity
Table 5. Masses and cooling times for WD0433+270 assuming a C/O and an Fe core.

\begin{tabular}{ccc}
\hline \hline Model & $M_{\mathrm{WD}}\left(M_{\odot}\right)$ & $t_{\text {cool }}(\mathrm{Gyr})$ \\
\hline C/O core & $0.67 \pm 0.03$ & $4.1 \pm 1.2$ \\
Fe core & $0.46 \pm 0.07$ & $1.0 \pm 0.1$ \\
\hline
\end{tabular}

of WD0433+270 to check whether it falls within the range of values corresponding to each set of cooling sequences. As expected, there is no correspondence with the $\mathrm{O} / \mathrm{Ne}$ - and He-core model sequences since the physics of white dwarfs with these compositions predicts masses for this radius that are too high or too low, respectively.

The most common internal structure of a white dwarf is thought to be a core made of $\mathrm{C} / \mathrm{O}$ surrounded by an $\mathrm{H}$-thick envelope on top of a He buffer, with compositions of $q(\mathrm{H})=$ $M_{\mathrm{H}} / M=10^{-4}$ and $q(\mathrm{He})=M_{\mathrm{He}} / M=10^{-2}$. The cooling sequences of Salaris et al. (2000) consider such a configuration, but with a larger abundance of $\mathrm{O}$ than $\mathrm{C}$ at the center of the core. These improved cooling sequences include an accurate treatment of the crystallization process of the $\mathrm{C} / \mathrm{O}$ core, including phase separation upon crystallization, together with state-of-theart input physics suitable for computing white dwarf evolution. As can be seen in Table 5, the cooling time derived ( $\sim$ Gyr) is in excellent agreement with the results obtained by Bergeron et al. (2001). This cooling time is 6 times longer than the age of the Hyades open cluster and 2 times longer than that of the older Hyades stream group - about 2 Gyr (Chereul et al. 1999). The assumption of an $\mathrm{O} / \mathrm{Ne}$-core white dwarf would give shorter cooling times, since the heat capacity of $\mathrm{O}$ and $\mathrm{Ne}$ is smaller than that of $\mathrm{C}$, and as a result white dwarfs of this type cool faster (by a factor of 2). We considered the cooling tracks of Althaus et al. (2007) for O/Ne-core white dwarfs (Fig. 2, top-right). The outer layer chemical stratification consists of a pure hydrogen envelope of $10^{-6} M_{\odot}$ overlying a helium-dominated shell of $4 \times$ $10^{-4} M_{\odot}$ and, below that, a buffer rich in ${ }^{12} \mathrm{C}$ and ${ }^{16} \mathrm{O}$. However, as mentioned before, this core composition does not reproduce a white dwarf with such a small radius.

In light of the recent results obtained by Kilic et al. (2007), who claim that He white dwarfs may exist in the Galaxy as a product of single stellar evolution, we also considered this composition. In Fig. 2 (bottom-left) we plotted the cooling sequences of Serenelli et al. (2002) for He-core white dwarfs. But, as in the case of the $\mathrm{O} / \mathrm{Ne}$-core models, they do not reproduce a white dwarf with this observed radius. Moreover, as can be seen in Fig. 2, the effective temperature and luminosity of WD0433+270 does not fall in the range of values covered by these tracks. In any case, He-core white dwarfs typically cool slower than $\mathrm{C} / \mathrm{O}$-core ones because of the larger heat capacity of $\mathrm{He}$ in comparison with $\mathrm{C}$ and $\mathrm{O}$. Although this is partly compensated for by the lower mass, the net result does not lead to shorter cooling times.

Finally, we have taken into account the cooling tracks of Panei et al. (2000b) for Fe-core white dwarfs, obtaining a cooling time of $\sim 1 \mathrm{Gyr}$, which is 4 times less than for $\mathrm{C} / \mathrm{O}$ white dwarfs. This is because $\mathrm{Fe}$ nuclei are much heavier than $\mathrm{C}$ or $\mathrm{O}$, hence, the specific heat per gram is much smaller. The cooling time derived is in much better agreement with the age of the Hyades open cluster when considering an Fe core. It should be noted that the cooling time of a white dwarf is just a lower limit to its total age, since the main-sequence lifetime of its progenitor, which is badly determined for single white dwarfs, should also be taken into account. However, this value 

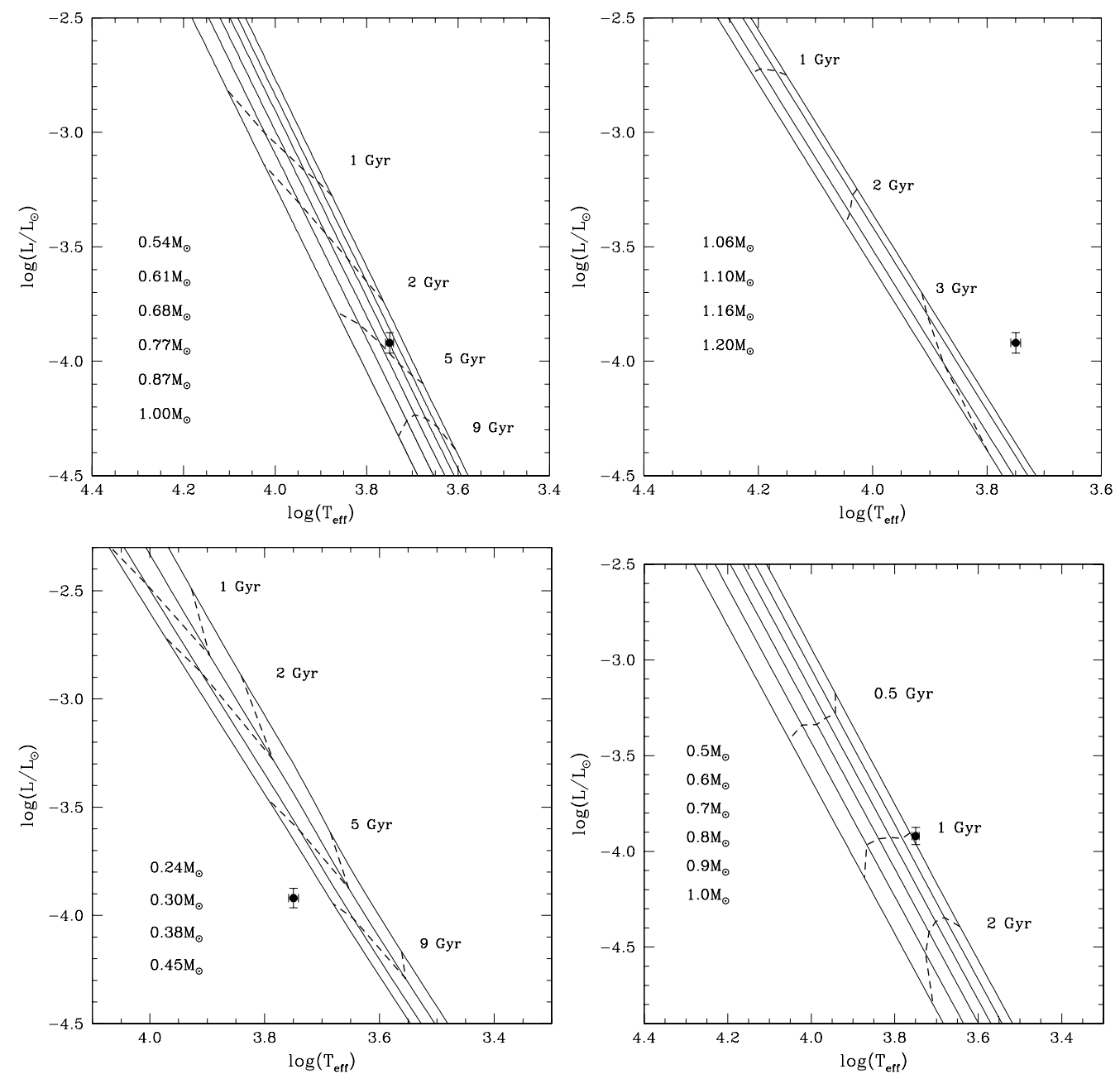

Fig. 2. Luminosity versus effective temperature for different cooling tracks: C/O-core (top-left), $\mathrm{O} / \mathrm{Ne}$-core (top-right), He-core (bottom-left), and Fe-core (bottom-right). The dashed lines represent cooling isochrones.

could be relatively low if the white dwarf progenitor is massive ( $\left.t_{\text {prog }} \ll 1 \mathrm{Gyr}\right)$. It is worth mentioning that the uncertainties in the cooling times are derived only from the observational parameters and do not consider a possible systematic contribution from the cooling sequences.

\section{Discussion and conclusions}

In this work we have presented new spectroscopic observations of the members of the common proper motion pair G39-27/289. Using these observations and the available photometry we have characterized these objects better, which helps us understand their nature.

Considering the kinematic properties of the members of the pair and the lithium detection in $\mathrm{BD}+26730$, we favor the scenario in which the common proper motion pair is indeed a former member of the Hyades cluster, and thus its members have a coeval age of $\sim 0.6-0.7$ Gyr. Having evaluated different compositions for WD0433+270, the young age inferred from cluster membership is only compatible with the case of an Fe core, which would have an associated cooling time of $\sim 1 \mathrm{Gyr}$. The agreement is not perfect, but modeling the cooling evolution of Fe-core white dwarfs could still have some associated uncertainties.

The existence of white dwarfs with an Fe-rich core has important consequences for the models of thermonuclear explosion of stellar degenerate cores. According to the theory of stellar evolution, all stars that develop an Fe core experience a collapse to a neutron star or a black hole, regardless of the mass loss rate assumed. However, there is still an alternative possibility of avoiding this final fate, which relies on the failure of the thermonuclear explosion of a degenerate white dwarf near the Chandrasekhar limit (Isern et al. 1991). Our current view of a thermonuclear explosion follows.

Once the thermonuclear runaway starts in the central regions of a white dwarf, the ignition front propagates outwards and injects energy at a rate that depends on the velocity at which matter is effectively burned leading to the expansion of the star. At the same time, electron captures on the incinerated matter efficiently remove energy at a rate determined by the density causing the contraction of the star. Therefore, depending on the ignition density and the velocity of the burning front, the outcome can be different. It is known that He-degenerate cores always explode, that $\mathrm{O} / \mathrm{Ne}$ and $\mathrm{Fe}$ degenerate cores always collapse, and that $\mathrm{C} / \mathrm{O}$-degenerate cores can explode or collapse depending on the ignition density. The existence of Fe-rich white dwarfs would imply the possibility of an intermediate behavior between those discussed above in which an Fe remnant is left after a mild explosion.

A detailed theory explaining the formation of Fe-core white dwarfs still needs to be developed, and up to now the possibility of their existence has been suggested mainly from observational 
evidence. Other examples of possible Fe-core white dwarfs have been found in the past. Provencal et al. (1998) singled out two objects whose radii and masses were too small to fit the typical C/O mass-radius relation. These authors analyzed a sample of white dwarfs, calculated their masses using either orbital parameters, gravitational redshifts, or spectroscopic fits, and determined their radii independently from the knowledge of effective temperatures and distances. Provencal et al. (1998) indicated that, in those two cases, a core made by Fe was the only plausible explanation that could account for their small radii.

Besides the Fe-core hypothesis, there is an alternative scenario that we cannot rule out. Although we think it is unlikely, the common proper motion pair might not be related in any way to the Hyades cluster. This would eliminate the age constraint and thus relax the requirement of an exotic composition for the white dwarf core. In this case, the age of the objects (both WD $0433+270$ and BD+26 730) could be $\sim 4$ Gyr. This would have some interesting consequences for our current view of star streams or moving groups in the Galaxy. As pointed out by Chereul et al. (1999), the Hyades stream contains at least three distinct age groups with ages up to 2 Gyr. The upper limit was probably a consequence of using $\mathrm{A}-\mathrm{F}$ stars as kinematic tracers, which would naturally have a cutoff age at about 2-3 Gyr. With an age of 4 Gyr, WD0433+270 and BD+26 730 would indicate the existence of an even older population in the Hyades stream, thus completely ruling out any coevality within the members. This would lend strong support to the model of a resonant origin for the Hyades stream (Famaey et al. 2007). Another implication of such an old age is the conflict with lithium detection, which would imply much lower destruction rate than expected.

Definitive proof supporting one of these scenarios will have to await an unambiguous determination of the mass of this object or, alternatively, a more conclusive study of its evolutionary link with the Hyades cluster.

Acknowledgements. We thank C. Allende Prieto for his help during the observations and his useful comments in the reduction and analysis of BD+26 730 . S. C. would like to acknowledge support from MEC through a FPU grant. This research was partially supported by the MEC grants AYA05-08013-C03-01 and 02, by the European Union FEDER funds and by the AGAUR.

\section{References}

Althaus, L. G., García-Berro, E., Isern, J., \& Córsico, A. H. 2005, A\&A, 441, 689

Althaus, L. G., García-Berro, E., Isern, J., Córsico, A. H., \& Rohrmann, R. D. 2007, A\&A, 465, 249
Barrado y Navascués, D., \& Stauffer, J. R. 1996, A\&A, 310, 879 Barrado y Navascués, D., Fernández-Figueroa, M. J., García López, R. J., De Castgro, E., \& Cornide, M. 1997, A\&A, 326, 780

Bergeron, P., Leggett, S. K., \& Ruiz, M. T. 2001, ApJS, 133, 413

Catalán, S., Isern, J., García-Berro, E., et al. 2007, A\&A, 477, 213

Cayrel, R., Hill, V., Beers, T. C., et al. 2001, Nature, 409, 691

Chereul, E., Crézé, M., \& Bienaymé, O. 1999, A\&ASS, 135, 5

de Bruijne, J. H. J. 1999, MNRAS, 306, 381

de Bruijne, J. H. J., Hoogerwerf, R., \& de Zeeuw, P. T. 2001, A\&A, 367, 111

Eggen, O. J. 1993a, AJ, 106, 642

Eggen, O. J. 1993b, AJ, 106, 1885

Eggen, O. J., \& Greenstein, J. L. 1965, ApJ, 141, 83

Famaey, B., Jorissen, A., Luri, X., et al. 2005, ESASP, 576, 129

Famaey, B., Pont, F., Luri, X., et al. 2007, A\&A, 461, 957

Fontaine, G., Brassard, P., \& Bergeron, P. 2001, PASP, 113, 409

Frebel, A., Christlieb, N., Norris, J. E., et al. 2007, ApJ, 660, 117

Holberg, J. B., Oswalt, T. D., \& Sion, E. M. 2002, ApJ, 571, 512

Isern, J., Canal, R., \& Labay, J. 1991, ApJ, 372, 83

Kilic, M., Stanek, K. Z., \& Pinsonneault, M. H. 2007, ApJ, submitted [arXiv:0706.3045]

Mamajek, E. E., Barrado y Navascués, D., Randich, S., et al. 2007, in Stellar Ages, Proceedings of the 14th Cambridge Workshop on Cool Stars, ed. G. van Belle [arXiv:astro-ph/0702074]

Masana, E., Jordi, C., \& Ribas, I. 2006, A\&A, 450, 735

Miglio, A., \& Montalbán, J. 2005, A\&A, 441, 615

Nordström, B., Mayor, M., Andersen, J., et al. 2004, A\&A, 418, 989

Oláh, K., Strassmeier, K. G., Kovári, Z., \& Guinan, E. F. 2001, A\&A, 372, 119

Oswalt, T., Hintzen, P., \& Luyten, W. 1988, ApJS, 66, 391

Oswalt, T. D., \& Strunk, D. 1994, BAAS, 26, 901

Panei, J. A., Althaus, L. G., \& Benvenuto, O. G. 2000a, A\&A, 353, 970

Panei, J. A., Althaus, L. G., \& Benvenuto, O. G. 2000b, MNRAS, 312, 531

Paunzen, E., \& Netopil, M. 2006, MNRAS, 371, 1641

Perryman, M. A. C., Lindegren, L., Kovalevsky, J., et al. 1997, A\&A, 323, 49

Perryman, M. A. C., Brown, A. G. A., Lebreton, Y., \& Gomez, A. 1998, A\&A, 331,81

Provencal, J. L., Shipman, H. L., Hog, E., \& Thejll, P. 1998, ApJ, 494, 759

Ribas, I., Jordi, C., Torra, J., \& Giménez, A. 2000, MNRAS, 313, 99

Salaris, M., García-Berro, E., Hernanz, M., Isern, J., \& Saumon, D. 2000, ApJ, 544,1036

Salim, S., \& Gould, A. 2003, ApJ, 582, 1011

Schultz, G., Zuckerman, B., \& Becklin, E. E. 1996, ApJ, 460, 402

Serenelli, A. M., Althaus, L. G., Rohrmann, R. D., \& Benvenuto, O. G. 2002, MNRAS, 337, 1091

Shipman, H. L., \& Provencal, J. L. 1999, in 11th European Workshop on White Dwarfs, ed. J. E. Solheim, \& E. G. Meistas, ASP Conf. Ser., 169, 15

Silvestri, N. M., Hawley, S. L., \& Oswalt, T. D. 2005, AJ, 129, 2428

Tokovinin, A., Thomas, S., Sterzik, M., \& Udry, S. 2006, A\&A, 450, 681

von Hippel, T., Simpson, C., \& Manset, N. 2001, in Astrophysical Ages and Times Scales, ed. T. von Hippel, C. Simpson, \& N. Manset, ASP Conf. Ser., 245,190

Wegner, G. 1973, MNRAS, 165, 271

Wood, M. A. 1995, in White Dwarfs, Proceedings of the 9th European Workshop on White Dwarfs, ed. D. Koester \& K. Werner (Berlin: Springer), Lecture Notes in Physics, 443, 51

Zuckerman, G., Koester, D., Reid, I. N., \& Hünsch, M. 2003, ApJ, 596, 477 